\title{
Prevalence of Non-Communicable Diseases among Retired Military Personnel
}

\author{
Khan $\mathrm{MMI}^{1}$, Haque $\mathrm{A}^{2}$, Shamimuzzaman $\mathrm{M}^{3}$, Nancy $\mathrm{C}^{4}$, Zafreen $\mathrm{F}^{5}$ \\ DOl:https://doi.org/10.3329/jafmc.v16i2.55295
}

\begin{abstract}
Introduction: Non-communicable diseases (NCDs) have become burning issues worldwide, especially among the elderly individuals. Bangladeshi elderly is also susceptible cohort to NCDs for its recent epidemiologic transition and the NCDs is casting as prominent risk for major mortality and morbidity related issues.
\end{abstract}

Objectives: To find out the prevalence of common NCDs and its risk factors among the Bangladeshi retired military personnel.

Methods: This cross-sectional study was conducted among the retired military personnel admitted in Combined Military Hospital (CMH), Savar from January 2019 to July 2019. A face-to-face interview was carried out among the respondents using a semi-structured questionnaire.

Results: Among 153 retired military personnel; most common NCDs were hypertension (80.4\%), diabetes mellitus $66.7 \%$, ischemic heart disease $37.3 \%$ and only $3.9 \%$ had low back pain. Most of them accessed their health in $\mathrm{CMH}(66.7 \%)$, where $17.6 \%$ reported to Govt. hospital and only a minor portion (15.7\%) to private hospital. Moreover, $70.6 \%$ of them were not willing to regular health check-up.

Conclusions: The most common NCDs were hypertension, diabetes mellitus and ischemic heart disease. This study concluded with a few intervention strategies and community-based health promotion programs to reduce the burden of NCDs among the retired military personnel.

Key-words: Non communicable diseases, Retired military personnel, Combined military hospital.

\section{Introduction}

According to World Health Organization ${ }^{1}$, non-communicable diseases (NCDs), also known as chronic diseases, tend to be of long duration diseases and are the result of a combination of genetic, physiological, environmental and behavioral factors. NCDs account for 41 million yearly deaths, equivalent to $71 \%$ of all deaths globally and disproportionately affect people in low and middle-income countries (such as Bangladesh, where the present study was carried out), where more than three quarters of global
NCD deaths (i. e. 32million) occurred ${ }^{1}$. Bangladesh is in the midst of an epidemiologic transition where the burden of disease is shifting from a disease profile dominated by infectious diseases, under-nutrition and conditions of childbirth to one increasingly characterized by NCDs, which accounts for $59 \%$ of total deaths (886,000 deaths) yearly ${ }^{2}$..

Now-a-days, with the increasement of life expectancy across worldwide over the past few decades, the proportion of elderly individuals has significantly increased (including Bangladesh; average life expectancy of Bangladeshi people rises to 72.49 years $)^{7,8}$. At present, the world accounts for approximately 900 million elderly people (aged $60+$ years) with a poll of $12 \%$ of total global population and it was sought to be this number as more than double (to two billion) by the year 2050 and of these $80 \%$ of these individuals will be living in low and middle-income countries such as Bangladesh $^{9}$. In Bangladesh ${ }^{10}$, about $7.7 \%$ of the total population are elderly, which accounts for 14 million population, which is estimated to increase to 17.2 million by 2025 . Along with the increase of elderly population portion, several physical and mental health consequences are related to age-specific suffering also increased among the elderly people ${ }^{3,7-12}$.

The retirement age for Bangladeshi public servants ${ }^{13}$, fixed after the country's independence, was originally set to 57 years, although the current govt. allows public servants to work till the age of 59, and public servants having a freedom fighters' certificate to work till 61. After the retirement of military personnel, not maintaining the disciplined and healthy life as did during service was sought. This situation mediating reasons were suspected to not having sufficient income and/or depending on others (i.e. son, daughter, wife etc.), being fully unemployed, having lack of calorie-rich food access etc., and these factors are sought after for developing $\mathrm{NCDs}^{7,14-16}$.

Based on recent Bangladeshi press reports, retired military individuals are suspected of suffering NCDs (where these co-morbid military individuals are less likely to be helpful to the Govt.), although there is no national statistics or any study relating to this cohorts' NCDs in the country. The situation reflects as there is a large knowledge gap in the country, where increased information would be helpful in preventive approaches and is possible to keep the military personnel

1. Col Md Mazharul Islam Khan, MBBS, MPH, Commandant, CMH, Savar (E-mail: mik100883@yahoo.com) 2. Maj Ariful Haque, MBBS, DA, FCPS, Classified Specialist in Anesthesiology, CMH, Savar 3. Capt Md Shamimuzzaman, MBBS, Medical Officer, CMH, Savar 4. Dr Chyochyo Nancy, MBBS, Medical Officer, OSD, DGHS, Mohakhali, Dhaka, 5. Dr Farzana Zafreen, MBBS, MPH, Associate Professor and Head, Department of Community Medicine, Medical College for Women \& Hospital, Uttara, Dhaka. 
fit for the hazardous circumstances of the country. Therefore, the present study investigated the prevalence of common NCDs and its socio-demographic predictors among the retired military individuals attending the $\mathrm{CMH}$, Savar.

\section{Materials and Methods}

This cross-sectional study was conducted among 153 retired military personnel admitted in Combined Military Hospital (CMH) Savar from January 2019 to July 2019. A face-to-face interview was carried out among the respondents using a semi-structured questionnaire. Informed consent was obtained from every participant. Confidentiality of data and privacy of the participants was strictly maintained. A purposive convenience sampling technique was utilized and data were analyzed using 22.0 version of SPSS. Descriptive statistics including (i.e., frequency, percentage etc.) and inferential statistics (i.e., correlation analysis) were carried out to assess the relationship between variables and NCDs. p-value $<0.05$ were regarded as significant for this study.

\section{Results}

Socio-demographic characteristics of 153 retired military personnel presented in Table-I. Most of them belong to $76+$ years age groups (45.1\%), followed by $60-65$ years (33.3\%), $71-75$ years (11.8\%) and $66-70$ years (9.8\%). About $54.9 \%$ of the participants were from rural part of the country, most of them were unemployed (54.9\%), only $39.2 \%$ of them used their morning time by jogging, $52.9 \%$ were smokers, and $62.7 \%$ belonged to nuclear family. Around $53 \%$ participants reported to have wife and children dependent on themselves, whereas $45.1 \%$ had only wife. Most of them got social supports from only family (82.4\%) and the main sources of their income were themselves (62.7\%). In consideration of socioeconomic status, majority of them belonged to middle class family (31.4\%), whereas $17.6 \%$ reported to be both lower and upper class. Prevalence of the common NCD showed in Figure-1; most of them were suffering from hypertension (80.4\%), whereas $66.7 \%$ from diabetes mellitus, $37.3 \%$ from ischemic heart disease and only $3.9 \%$ from low back pain. Respondents' access to the health care facilities showed; most of them accessed their health in $\mathrm{CMH}$ (66.7\%), where $17.6 \%$ reported to Govt. hospital and only a minor portion (15.7\%) to private hospital. About $70.6 \%$ of them were not willing to go for regular health care assessment. Retired military personnel's hypertension status was significantly $(p<0.05)$ associated with socio-demographic factors like; socio-economic status, duration of suffering and regular health checkup status. Status of Diabetes Mellitus was significantly $(p<0.05)$ associated with socio-demographic factors like; type of permanent residence, duration of retired life, use of morning time, smoking status, family type, type of food intake, BMI, duration of suffering, type of hospital used and regular health checkup. Ischemic heart disease status was significantly $(p<0.05)$ associated with socio-demographic factors like; age group, present occupation, year of retired life, smoking status, dependents, financial dependency, food intake type, BMI, duration of sufferings and health care type (Table-II).
Table-I: Distribution of respondents by the socio-demographic variables $(n=153)$

\begin{tabular}{|c|c|c|c|}
\hline \multicolumn{2}{|c|}{ Variables } & Frequency & Percentage \\
\hline \multirow{4}{*}{ Age group } & $60-65$ years & 51 & 33.3 \\
\hline & 66-70 years & 15 & 9.8 \\
\hline & $71-75$ years & 18 & 11.8 \\
\hline & $76+$ years & 69 & 45.1 \\
\hline \multirow{2}{*}{$\begin{array}{l}\text { Permanent } \\
\text { residence }\end{array}$} & Rural & 69 & 45.1 \\
\hline & Urban & 84 & 54.9 \\
\hline \multirow{2}{*}{$\begin{array}{l}\text { Educational } \\
\text { qualification }\end{array}$} & SSC & 90 & 58.8 \\
\hline & $\mathrm{HSC}$ & 54 & 35.3 \\
\hline \multirow{3}{*}{$\begin{array}{l}\text { Present } \\
\text { occupation }\end{array}$} & Unemployment & 84 & 54.9 \\
\hline & Business & 33 & 21.6 \\
\hline & Service & 36 & 23.5 \\
\hline \multirow{4}{*}{$\begin{array}{l}\text { Year of retired } \\
\text { life }\end{array}$} & More than 15 years & 45 & 29.4 \\
\hline & 10 to 15 years & 36 & 23.5 \\
\hline & 5 to 10 years & 30 & 19.6 \\
\hline & Less than 5 years & 39 & 25.5 \\
\hline \multirow{3}{*}{$\begin{array}{l}\text { Use of morning } \\
\text { time }\end{array}$} & Sleeping & 18 & 11.8 \\
\hline & Prayer & 75 & 49.0 \\
\hline & Jogging & 60 & 39.2 \\
\hline \multirow[t]{2}{*}{ Smoking status } & Yes & 81 & 52.9 \\
\hline & No & 72 & 47.1 \\
\hline \multirow{3}{*}{$\begin{array}{l}\text { Family } \\
\text { members }\end{array}$} & Less than 5 & 30 & 19.6 \\
\hline & 6 to 7 & 75 & 49.0 \\
\hline & More than 7 & 36 & 23.5 \\
\hline \multirow[t]{2}{*}{ Family type } & Nuclear & 96 & 62.7 \\
\hline & Joint & 54 & 35.3 \\
\hline \multirow[t]{2}{*}{ Dependents } & Wife and children & 81 & 52.9 \\
\hline & Only wife & 69 & 45.1 \\
\hline \multirow[t]{2}{*}{ Social support } & Only family & 126 & 82.4 \\
\hline & $\begin{array}{l}\text { Family, friends and } \\
\text { relatives }\end{array}$ & 27 & 17.6 \\
\hline \multirow{3}{*}{$\begin{array}{l}\text { Socio-economic } \\
\text { status }\end{array}$} & Lower class & 27 & 17.6 \\
\hline & Middle class & 48 & 31.4 \\
\hline & Higher class & 27 & 17.6 \\
\hline \multirow{2}{*}{$\begin{array}{l}\text { Financial } \\
\text { Dependence }\end{array}$} & Son & 57 & 37.3 \\
\hline & Self & 96 & 62.7 \\
\hline \multirow{2}{*}{$\begin{array}{l}\text { Food intake } \\
\text { type }\end{array}$} & High calorie & 9 & 5.9 \\
\hline & Low calorie & 144 & 94.1 \\
\hline
\end{tabular}

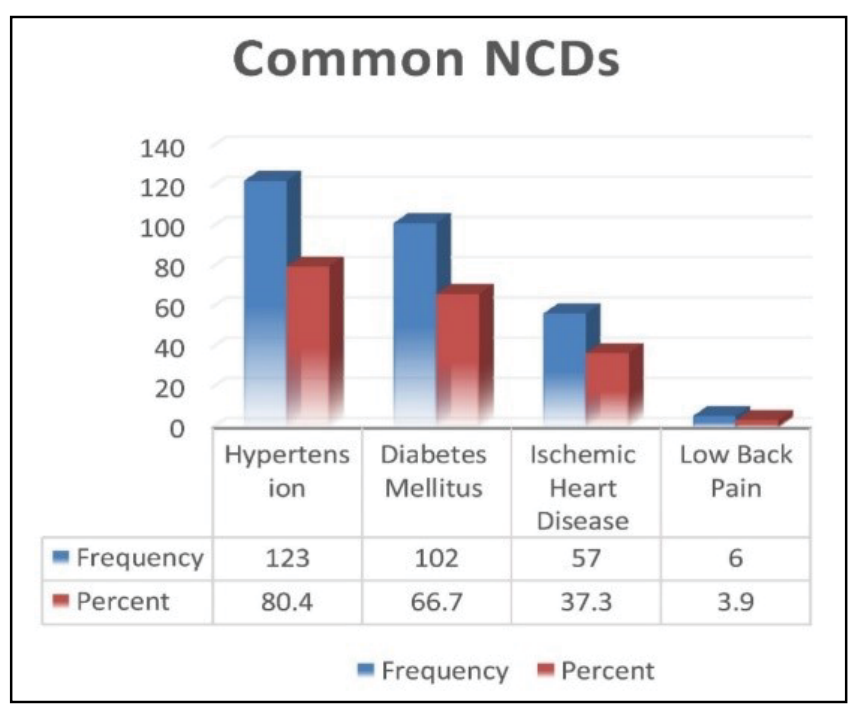

Figure-1: Distribution of common NCDs ( $n=153)$ 
Table-II: Association between socio-demographics and health information related variables with Hypertension, Diabetes Mellitus and Ischemic Heart Disease ( $n=153)$

\begin{tabular}{|c|c|c|c|c|c|c|c|c|c|c|}
\hline \multicolumn{2}{|c|}{$\begin{array}{l}\text { Variables } \\
\end{array}$} & \multicolumn{3}{|c|}{ Hypertension } & \multicolumn{3}{|c|}{ Diabetes Mellitus } & \multicolumn{3}{|c|}{ Ischemic Heart Disease } \\
\hline \multirow{5}{*}{$\begin{array}{l}\text { Age group } \\
\text { years }\end{array}$} & & & & \multirow{5}{*}{$\begin{array}{l}x^{2}=4.536 \\
d f=3 \\
p>0.05\end{array}$} & & & \multirow{5}{*}{$\begin{array}{l}x^{2}=6.699 \\
d f=3 \\
p>0.05\end{array}$} & & & \multirow{5}{*}{$\begin{array}{l}X^{2}=10.054 \\
d f=3 \\
p<0.05\end{array}$} \\
\hline & $60-65$ & $39(76.5)$ & $12(23.5)$ & & $39(76.5)$ & $12(23.5)$ & & $27(52.9)$ & $24(47.1)$ & \\
\hline & $66-70$ & $12(80)$ & $3(20.0)$ & & $12(80.0)$ & $3(20.0)$ & & $6(40.0)$ & $9(60.0)$ & \\
\hline & $71-75$ & $12(66.7)$ & $6(33.3)$ & & $9(50.0)$ & $9(50.0)$ & & $3(16.7)$ & $15(83.3)$ & \\
\hline & $\geq 76$ & $60(87.0)$ & $9(13.0)$ & & $42(60.9)$ & $27(39.1)$ & & $21(30.4)$ & $48(69.6)$ & \\
\hline \multirow{2}{*}{$\begin{array}{l}\text { Permanent } \\
\text { residence }\end{array}$} & Rural & $57(82.6)$ & $12(17.4)$ & \multirow{2}{*}{$\begin{array}{l}x^{2}=0.392 \\
d f=1 \\
p>0.05\end{array}$} & $39(56.5)$ & $30(43.5)$ & \multirow{2}{*}{$\begin{array}{l}X^{2}=5.821 \\
d f=1 \\
p<0.05\end{array}$} & $21(30.4)$ & $48(69.6)$ & \multirow{2}{*}{$\begin{array}{l}X^{2}=2.501 \\
d f=1 \\
p>0.05\end{array}$} \\
\hline & Urban & 66 (78.6) & $18(21.4)$ & & $63(75.0)$ & $21(25.0)$ & & $36(42.9)$ & $48(57.1)$ & \\
\hline \multirow{2}{*}{$\begin{array}{l}\text { Education } \\
\text { status }\end{array}$} & $\leq \mathrm{SSC}$ & $75(83.3)$ & $15(16.7)$ & \multirow{2}{*}{$\begin{array}{l}x^{2}=2.526 \\
d f=1 \\
p>0.05\end{array}$} & $54(60.0)$ & $36(40.0)$ & \multirow{2}{*}{$\begin{array}{l}x^{2}=2.204 \\
d f=1 \\
p>0.05\end{array}$} & $27(30.0)$ & $63(70.0)$ & \multirow{2}{*}{$\begin{array}{l}X^{2}=3.079 \\
d f=1 \\
p>0.05\end{array}$} \\
\hline & $\geq \mathrm{HSC}$ & $39(72.2)$ & $15(27.8)$ & & $39(72.2)$ & $15(27.8)$ & & $24(44.4)$ & $30(55.6)$ & \\
\hline \multirow{3}{*}{$\begin{array}{l}\text { Present } \\
\text { occupation }\end{array}$} & Unemployed & 66 (78.6) & $18(21.4)$ & \multirow{3}{*}{$\begin{array}{l}X^{2}=0.417 \\
d f=2 \\
p>0.05\end{array}$} & $54(64.3)$ & $30(35.7)$ & \multirow{3}{*}{$\begin{array}{l}X^{2}=0.760 \\
d f=2 \\
p>0.05\end{array}$} & $39(46.4)$ & $45(53.6)$ & $x^{2}=8.397$ \\
\hline & Business & $27(81.8)$ & $6(18.2)$ & & $24(72.7)$ & $9(27.3)$ & & $6(18.2)$ & $27(81.8)$ & $\mathrm{df}=2$ \\
\hline & Service & $30(83.3)$ & $6(16.7)$ & & $24(66.7)$ & $12(33.3)$ & & $12(33.3)$ & $24(66.7)$ & $p<0.05$ \\
\hline Year of retired & $<5$ & $27(69.2)$ & $12(30.8)$ & $x^{2}=5.889$ & $18(46.2)$ & $21(53.8)$ & $x^{2}=22.583$ & $18(46.2)$ & $21(53.8)$ & $x^{2}=16.136$ \\
\hline & 5 to 10 & $24(80.0)$ & $6(20.0)$ & $\mathrm{df}=3$ & $27(90.0)$ & $3(10.0)$ & $\mathrm{df}=3$ & $12(40)$ & $18(60)$ & $\mathrm{df}=3$ \\
\hline & 10 to 15 & $33(91.7)$ & $3(8.3)$ & $p>0.05$ & $30(83.3)$ & $6(16.7)$ & $p<0.001$ & $3(8.3)$ & 33 (91.7) & $p<0.001$ \\
\hline & $>15$ & $36(80.0)$ & $9(20.0)$ & & $24(53.3)$ & $21(46.7)$ & & $21(46.7)$ & $24(53.3)$ & \\
\hline Use of morning & Sleeping & $15(83.3)$ & $3(16.7)$ & $x^{2}=0.112$ & $9(50.0)$ & $9(50.0)$ & $x^{2}=49.590$ & $9(50.0)$ & $9(50.0)$ & $x^{2}=2.844$ \\
\hline time & Prayer & $60(80.0)$ & $15(20.0)$ & $d f=2$ & $33(44.0)$ & $42(56.0)$ & $d f=2$ & $30(40.0)$ & $45(60.0)$ & \\
\hline & Jogging & $48(80.0)$ & $12(20.0)$ & $p>0.05$ & $60(100)$ & $0(0.0)$ & $p<0.001$ & $18(30.0)$ & $42(70.0)$ & $p>0.05$ \\
\hline Smoking status & Yes & $69(85.2)$ & $12(14.8)$ & $x^{2}=2.509$ & $48(59.3)$ & $33(40.7)$ & $x^{2}=4.250$ & $24(29.6)$ & $57(70.4)$ & $x^{2}=4.281$ \\
\hline & No & $54(75.0)$ & $18(25.0)$ & $\begin{array}{l}d f=1 \\
p>0.05\end{array}$ & $54(75.0)$ & $18(25.0)$ & $\begin{array}{l}\mathrm{df}=1 \\
\mathrm{p}<0.05\end{array}$ & $33(45.8)$ & 39 (54.2) & $\begin{array}{l}\mathrm{df}=1 \\
\mathrm{p}<0.05\end{array}$ \\
\hline $\begin{array}{l}\text { Family } \\
\end{array}$ & $<5$ & $30(83.3)$ & $6(16.7)$ & $x^{2}=0.818$ & $24(80.0)$ & $6(20.0)$ & $x^{2}=5.008$ & $12(33.3)$ & $24(66.7)$ & $x^{2}=2.282$ \\
\hline members & 6 to 7 & $57(76.0)$ & $18(24.0)$ & $d f=2$ & $45(60.0)$ & $30(40.0)$ & $d f=2$ & $33(44.0)$ & $42(56.0)$ & \\
\hline & $>7$ & $24(80.0)$ & $6(20.0)$ & $p>0.05$ & $24(80.0)$ & $6(20.0)$ & & $9(30.0)$ & $21(70.0)$ & \\
\hline Family type & Nuclear & $78(81.3)$ & $18(18.8)$ & $x^{2}=0.260$ & $57(59.4)$ & $39(40.6)$ & $x^{2}=9.116$ & $39(40.6)$ & $57(59.4)$ & $x^{2}=0.780$ \\
\hline & Joint & $42(77.8)$ & $12(22.2)$ & $\begin{array}{l}\mathrm{df}=1 \\
p>0.05\end{array}$ & $45(83.3)$ & $9(16.7)$ & $\begin{array}{l}\mathrm{df}=1 \\
\mathrm{p}<0.01\end{array}$ & $18(33.3)$ & $36(66.7)$ & $\begin{array}{l}\mathrm{df}=1 \\
p>0.05\end{array}$ \\
\hline Dependents & $\begin{array}{l}\text { Wife and } \\
\text { children }\end{array}$ & $57(82.6)$ & $12(17.4)$ & $\begin{array}{l}x^{2}=0.032 \\
d f=1\end{array}$ & $57(70.4)$ & $24(29.6)$ & $\begin{array}{l}x^{2}=1.499 \\
d f=1\end{array}$ & $33(47.8)$ & 36 (52.2) & $\begin{array}{l}x^{2}=5.237 \\
d f=1\end{array}$ \\
\hline & Only wife & 66 (81.5) & $15(18.5)$ & $p>0.05$ & $42(60.9)$ & $27(39.1)$ & $p>0.05$ & $24(29.6)$ & 57 (70.4) & $p<0.05$ \\
\hline Social support & $\begin{array}{l}\text { Family, friends } \\
\text { and relatives }\end{array}$ & $21(77.8)$ & $6(22.2)$ & $\begin{array}{l}X^{2}=0.142 \\
d f=1\end{array}$ & $15(55.6)$ & $12(44.4)$ & $\begin{array}{l}X^{2}=1.821 \\
d f=1\end{array}$ & $9(33.3)$ & $18(66.7)$ & $\begin{array}{l}X^{2}=0.216 \\
d f=1\end{array}$ \\
\hline & Family & $102(81.0)$ & $24(19.0)$ & $p>0.05$ & $87(69.0)$ & $39(31.0)$ & $p>0.05$ & 48 (38.1) & $78(61.9)$ & $p>0.05$ \\
\hline Socio- & Lower & $24(88.9)$ & $3(11.1)$ & $x^{2}=6.304$ & $18(66.7)$ & $9(33.3)$ & $x^{2}=3.001$ & $12(44.4)$ & $15(55.6)$ & $x^{2}=1.379$ \\
\hline economic class & Middle & $33(68.8)$ & $15(31.3)$ & $\mathrm{df}=2$ & $36(75.0)$ & $12(44.4)$ & $d f=2$ & $15(31.3)$ & $33(68.8)$ & $d f=2$ \\
\hline & Higher & $24(88.9)$ & $3(11.1)$ & $p<0.05$ & $15(55.6)$ & $12(44.4)$ & $p>0.05$ & $9(33.3)$ & $18(66.7)$ & $p>0.05$ \\
\hline Financial & Son & 45 (78.9) & $12(21.1)$ & $x^{2}=0.120$ & $39(68.4)$ & $18(31.6)$ & $x^{2}=0.126$ & $27(47.4)$ & $30(52.6)$ & $x^{2}=3.975$ \\
\hline dependence & Self & 78 (81.3) & $18(18.8)$ & $\begin{array}{l}d f=1 \\
p>0.05\end{array}$ & $63(65.6)$ & $33(34.4)$ & $\begin{array}{l}d f=1 \\
p>0.05\end{array}$ & $30(31.3)$ & $66(62.7)$ & $\begin{array}{l}\mathrm{df}=1 \\
\mathrm{p}<0.05\end{array}$ \\
\hline Food intake & Low calorie & $114(79.2)$ & $30(19.6)$ & $x^{2}=2.332$ & $99(68.8)$ & $45(31.3)$ & $x^{2}=4.781$ & $48(33.3)$ & $96(66.7)$ & $x^{2}=16.105$ \\
\hline & High calorie & $9(100)$ & $0(0)$ & $\begin{array}{l}d f=1 \\
p>0.05\end{array}$ & $3(33.3)$ & $6(66.7)$ & $\begin{array}{l}d f=1 \\
p<0.05\end{array}$ & $9(100.0)$ & $0(0.0)$ & $\begin{array}{l}d f=1 \\
p<0.001\end{array}$ \\
\hline Body Mass & Overweight & $84(80.0)$ & $21(20.0)$ & $x^{2}=0.033$ & $81(77.1)$ & $24(22.9)$ & $x^{2}=16.529$ & $45(42.9)$ & $60(57.1)$ & $x^{2}=4.494$ \\
\hline & Normal & $39(81.3)$ & $9(18.8)$ & $\begin{array}{l}\hat{d f}=1 \\
p>0.05\end{array}$ & $21(43.8)$ & $27(56.3)$ & $\begin{array}{l}\hat{d f}=1 \\
p<0.001\end{array}$ & $12(25.0)$ & $36(75.0)$ & $\begin{array}{l}\hat{d f}=1 \\
p<0.05\end{array}$ \\
\hline Sufferings & $>15$ & $36(80.0)$ & $9(20.0)$ & $x^{2}=24.309$ & $27(60.0)$ & $18(40.0)$ & $x^{2}=10.286$ & $21(46.7)$ & $24(53.3)$ & $x^{2}=14.883$ \\
\hline & 11 to 15 & $36(85.7)$ & $6(14.3)$ & & $30(71.4)$ & $12(28.6)$ & & $6(14.3)$ & $36(85.7)$ & \\
\hline years & 5 to 10 & $42(93.3)$ & $3(6.7)$ & $p<0.001$ & $36(80.0)$ & $9(20.0)$ & $p<0.05$ & $18(40.0)$ & $27(60.0)$ & $p<0.01$ \\
\hline & $<5$ & $9(42.9)$ & $12(57.1)$ & & $9(42.9)$ & $12(57.1)$ & & $12(57.1)$ & $9(42.9)$ & \\
\hline Type of & Private & $20(83.3)$ & $4(16.7)$ & $x^{2}=0.249$ & $18(75.0)$ & $6(25.0)$ & $x^{2}=7.456$ & $6(25.0)$ & $18(75.0)$ & $x^{2}=6.207$ \\
\hline hospital used & Government & $21(77.8)$ & $6(22.2)$ & $d f=2$ & $12(44.4)$ & $15(55.6)$ & $d f=2$ & $6(22.2)$ & $21(77.8)$ & \\
\hline & $\mathrm{CMH}$ & $82(80.4)$ & $20(19.6)$ & $p>0.05$ & $72(70.6)$ & $30(29.4)$ & $p<0.05$ & $45(44.1)$ & $57(55.9)$ & $p<0.05$ \\
\hline Regular health & No & $81(75.0)$ & $27(25.0)$ & $x^{2}=6.773$ & 66 (61.1) & $42(38.9)$ & $x^{2}=5.100$ & $42(38.9)$ & 66 (61.1) & $x^{2}=0.419$ \\
\hline & Yes & 42 (93.3) & $3(6.7)$ & $\begin{array}{l}d f=1 \\
p<0.01\end{array}$ & $36(80.0)$ & $9(20.0)$ & $\begin{array}{l}d f=1 \\
p<0.05\end{array}$ & $15(33.3)$ & $30(66.7)$ & $\begin{array}{l}d f=1 \\
p>0.05\end{array}$ \\
\hline
\end{tabular}

\section{Discussion}

NCDs disproportionately affect people in low and middle-income countries where more than three quarters of global NCD deaths (32million) occur. Globally, cardiovascular diseases account for most NCD deaths (17.9 million people annually), followed by cancers (9.0 million), respiratory diseases (3.9million) and diabetes (1.6 million) $)^{1}$, where Bangladeshi elderly died by $18.9 \%$ for stroke or cardio-vascular accident and followed by chronic obstructive pulmonary disease $(16.8 \%)$, cancer $(7.7 \%)$ etc ${ }^{17}$. This study revealed the most sought after NCDs to be hypertension (80.4\%), and followed by $66.7 \%$ diabetes mellitus, and $37.3 \%$ ischemic heart disease. Compared to previous Bangladeshi study conducted among elderly individuals showed lower level of prevalence rates i.e., $12.6 \%$ hypertension, $2.8 \%$ diabetes mellitus, $9.3 \%$ chronic obstructive pulmonary disease etc ${ }^{17}$ and $38.7 \%$ hypertension, $6.9 \%$ chronic obstructive pulmonary disease, $2.4 \%$ heart disease ${ }^{7}$. However, a recent study from China showed the elderly were 
suffering as $48.0 \%$ hypertension, $21.9 \%$ diabetes, $14.2 \%$ cataract (although we didn't get any report in our survey suffering from cataract), $11.2 \%$ coronary heart disease, $6.8 \%$ stroke, $2.6 \%$ cancer ${ }^{14}$.

Therefore, from this finding (as well as previous Bangladeshi findings) hypertension is considered the most vulnerable disease for Bangladeshi elderly people and it accounts for most of the elderly deaths. However, the detection, treatment and control of hypertension are considered to be inadequate in many high-income countries; in low-income countries, where even consciousness about hypertension is generally low, the situation is even worse ${ }^{5}$. A recent study in the Netherland revealed only one third of the individuals with hypertension were aware of their condition and among those about $60 \%$ were treated but only $42 \%$ had their blood pressure well controlled ${ }^{18}$. Another study in China reported only $31 \%$ hypertensive people were treated and only 20\% were controlled $^{19}$. In the United States, $65 \%$ of hypertensive individuals received treatment and nearly $50 \%$ were controlled ${ }^{20}$. Herein, we suggest to increase health education and promotion related program (along with clinical facilities) in the country for not only hypertension reduction but also other NCDs. Although the government has initiated many NCD-related policies or programs, they lacked proper planning, implementation and monitoring. As a result, Bangladesh over the years had little success in effectively addressing the growing burden of non-communicable diseases. It is imperative that future research will critically assess the effectiveness of national NCD policies by monitoring their implementation and level of population coverage ${ }^{6}$.

Moreover, this study showed a wide ranges of socio-demographics risk factors related to NCDs development. Globally, it was sought over 7.2 million deaths every year due to tobacco and it is projected to increase markedly over the coming years. Moreover, 4.1 million annual deaths have been attributed to excess salt/sodium intake (with having no proper dietary habit), more than half of the 3.3 million annual deaths attributable to alcohol use are from NCDs and 1.6 million deaths annually can be attributed to insufficient physical activity ${ }^{1}$. For reducing the major risk factors for noncommunicable diseases - tobacco use, physical inactivity, unhealthy diet and the harmful use of alcohol is the focus of WHO's work to prevent deaths from NCDs ${ }^{15-16}$. Another concerning issue found in this study is $70.6 \%$ of the military personnel don't go for regular health care assessment. But, regular health checkup can help to find out problems before it affects harmfully. By getting the right health services, screenings, and treatments, will help to increase the chances for living a longer, healthier life. Therefore, it is recommended to make available a cost-effective health care access for Bangladeshi individuals. The generalizability of findings in the present study are limited by the cross-sectional nature of the data, the small sample size, and the non-representative sample from $\mathrm{CMH}$, Savar.

\section{Conclusion}

The results of our study showed the most common health problems were hypertension, diabetes mellitus and ischemic heart disease among the retired military personnel who were admitted in Combined Military Hospital, Savar. A wide range of socio-demographic and health related variables are associated with these sufferings. Military personnel are reservist of the govt., but they are ought to join in the team anytime if the country requires. But this study doesn't support if they are capable to do as NCDs are prevalent to them. So, the clear picture having lost majority of man power of the government as any NCDs affected won't be capable to go forward on fire as well as other emergency circumstances when needed. Hence, intervention strategies and community-based health promotion programs to reduce the burden of NCDs among the older population are badly suggested.

\section{References}

1. WHO. Noncommunicable diseases. https://www.who.int/news-room/fact-sheets/detail/-noncommunicablediseases. Published 2019. Accessed August 28, 2019.

2. icddrb. Non-communicable diseases. https://www.icddrb.org/ news-and-events/press-corner/media-resources/non-communicablediseases. Published 2019. Accessed August 28, 2019.

3. Kabir SMH, Islam MZ, Begum M et al. Health problems of elderly patients attending Combined Military Hospital, Dhaka. J Armed Forces Med Coll Bangladesh. 2017;13(1):8-12.

4. Bleich SN, Koehlmoos TLP, Rashid M et al. Noncommunicable chronic disease in Bangladesh: overview of existing programs and priorities going forward. Health Policy. 2011;100(2-3):282-289.

5. Khanam MA, Lindeboom W, Koehlmoos TLP et al. Hypertension: adherence to treatment in rural Bangladesh-findings from a population-based study. Glob Health Action. 2014;7(1):25028.

6. Biswas T, Pervin S, Tanim MIA et al. Bangladesh policy on prevention and control of non-communicable diseases: A policy analysis. BMC Public Health. 2017;17(1):582.

7. Khanam MA, Streatfield PK, Kabir ZN et al. Prevalence and patterns of multimorbidity among elderly people in rural Bangladesh: A cross-sectional study. J Health Popul Nutr. 2011;29(4):406.

8. Islam MA, Rahman MF. Health problems of elderly people in Bangladesh. J Armed Forces Med Coll Bangladesh. 2017;13(1):1-2.

9. WHO. Ageing and health. https://www.who.int/news-room/fact-sheets/detail/ageing-and-health. Published 2018. Accessed June 10, 2019.

10. Bangladesh Bureau of Statistics. Elderly Population in Bangladesh: Current Features and Future Perspectives. Dhaka, BD; 2015. http://203.112.218.65:8008/WebTestApplication/user files/Image/-PopMonographs/elderlyFinal.pdf. Published 2015. Accessed August 28, 2019.

11. Barikdar A, Ahmed T, Lasker SP. The situation of the elderly in Bangladesh. Bangladesh J Bioeth. 2016;7(1):27-36. 
12. Disu TR, Anne NJ, Griffiths M et al. Risk factors of geriatric depression among elderly Bangladeshi people: A pilot interview study. Asian J Psychiatr. 2019;44:163-169.

13. Dhaka Tribune. Parliamentary panel for increasing age limit for govt jobs to 35, retirement 65. https://www.dhakatribune.com/ bangladesh/2018/06/27/parliamentary-panel-for-increasingage-limit-for-govt-jobs-to-35-retirement-65. Published 2018. Accessed August 28, 2019.

14. Gu J, Chao J, Chen W et al. Multimorbidity in the communitydwelling elderly in urban China. Arch Gerontol Geriatr. 2017; 68:62-67.

15. Agborsangaya $C B$, Lau $D$, Lahtinen $M$ et al. Multimorbidity prevalence and patterns across socioeconomic determinants: A cross-sectional survey. BMC Public Health. 2012;12(1):201.

16. Ha NT, Le NH, Khanal V, Moorin R. Multimorbidity and its social determinants among older people in southern provinces, Vietnam.
Int J Equity Health. 2015;14(1):50..

17. Hosain GMM, Begum A. Health needs and health status of the elderly in rural Bangladesh. Asia Pac J Public Heal. 2003;15(1):3-9.

18. Scheltens T, Bots ML, Numans ME et al. Awareness, treatment and control of hypertension: the 'rule of halves' in an era of risk-based treatment of hypertension. J Hum Hypertens. 2007;21(2):99.

19. Wu $\mathrm{Y}$, Huxley $\mathrm{R}$, Li $\mathrm{L}$ et al. Prevalence, awareness, treatment, and control of hypertension in China: data from the China National Nutrition and Health Survey 2002. Circulation. 2008;118(25):2679-2686.

20. Ong KL, Cheung BMY, Man YB et al. Prevalence, awareness, treatment, and control of hypertension among United States adults 1999-2004. Hypertension. 2007;49(1):69-75. 